\title{
Cardiovascular Disease in Women-Challenges Deserving a Comprehensive Translational Approach
}

\author{
Jennifer S. Lee • Klea Bertakis • Frederick J. Meyers • \\ Erica Chedin • Alice Tarantal • Kent Anderson • \\ Lars Berglund
}

Received: 13 March 2009/Accepted: 5 May 2009 /Published online: 20 May 2009

(C) The Author(s) 2009. This article is published with open access at Springerlink.com

\begin{abstract}
Heart disease in women is associated with high levels of morbidity and mortality. Although many of the underlying causes are similar for both genders, cardiovascular disease among women has some unique features, including higher coronary heart disease mortality, higher frequency of sudden cardiac death without previous symptoms, and increased mortality among older women
\end{abstract}

J. S. Lee $\cdot$ F. J. Meyers $\cdot$ K. Anderson $\cdot$ L. Berglund $(\bowtie)$

Department of Medicine, University of California, Davis, UC

Davis Medical Center, CTSC,

2921 Stockton Blvd, Suite 1400,

Sacramento, CA 95817, USA

e-mail: lars.berglund@ucdmc.ucdavis.edu

\section{K. Bertakis}

Department of Family Medicine, University of California Davis, Sacramento, CA, USA

\section{A. Tarantal}

Department of Pediatrics, University of California Davis,

Sacramento, CA, USA

\section{A. Tarantal}

Department of Cell Biology and Human Anatomy,

University of California Davis,

Sacramento, CA, USA

F. J. Meyers · E. Chedin · A. Tarantal $\cdot$ K. Anderson $\cdot$ L. Berglund Clinical and Translational Science Center,

University of California Davis,

Sacramento, CA, USA

A. Tarantal

California National Primate Research Center,

University of California Davis,

Sacramento, CA, USA

L. Berglund

VA Northern California Health Care System,

Sacramento, CA, USA compared to men following a myocardial infarction. During recent years, increasing efforts have been placed on identifying preventive measures, but translation of knowledge from epidemiological studies and clinical trials remain incomplete, particularly in women. The recent launch of the National Institutes of Health's Clinical and Translational Science Award program offers opportunities to address these gaps and represent a unique opportunity to foster a new generation of researchers familiar with important issues regarding women's cardiovascular health.

Keywords Heart Disease · Informatics · Education ·

Translational Research

\section{Introduction}

Heart disease is an important contributor to morbidity and mortality among women. While many of the underlying cardiovascular risk factors are similar for both women and men, cardiovascular disease among women has some unique features. These include a higher frequency of women with sudden death without previous symptoms and an increased mortality among older women compared to men following a myocardial infarction [1-3]. These factors may contribute to higher coronary heart disease mortality in women compared to men, a fact not commonly recognized by physicians [1, 4]. During recent years, increasing efforts have been placed on identifying preventive measures, and in 2007, an update on evidence-based guidelines for cardiovascular disease prevention in women was published [3]. However, sufficient translation of knowledge from epidemiological studies and clinical trials into clinical practice is lacking, particularly among women who might be unaware that they are at risk. The recent 
launch of the National Institutes of Health's Clinical and Translational Science Award (CTSA) program offers opportunities to address these gaps. The CTSA program has already had a profound impact on clinical research and training methods related to women's health issues at the University of California, Davis (UC Davis) [5-7].

UC Davis was among the first 12 institutions to receive $\mathrm{NIH}$ funding for a CTSA and created its Clinical and Translational Science Center (CTSC) in 2006 [7, 8]. The funding accelerated and further integrated an existing conscientious planning effort for translational research with a stepwise approach to gradually increase our institutional competencies, capabilities, and resources in this area. The development of our CTSC has led us to develop new ways of bringing together a diverse faculty and facilitating research. In particular, the CTSC has impacted on our research training programs, providing a pathway to fully integrate trainees across the translational spectrum.

\section{Cardiovascular Disease in Women-Current Issues}

Although considerable progress has been made for disease prevention as well as therapeutic intervention in the area of cardiovascular health, heart disease remains a particular challenge for women. Women have a higher mortality rate after an initial myocardial infarction than men, and the majority of women suffering from an acute cardiovascular event do not make a full recovery. Further, the vast majority of middle-aged women have at least one risk factor for heart disease [2]. Although cardiovascular disease can, to a large extent, be prevented by attention to established risk factors, many women are unaware that they are at risk [9]. Assessment of cardiovascular risk in the USA is firmly based on application of the Framingham risk score system, and this system often underestimates the actual cardiovascular risk in women [10]. In addition, a recent study demonstrated a higher relative risk for coronary heart disease related to the commonly occurring metabolic syndrome among women compared to men [11]. Further, the nature of disease manifestations as well as clinical symptoms may differ across gender. Thus, among women, the atherosclerotic process is more distributed in the vascular tree, and symptoms such as fatigue, dyspnea, and shoulder or neck pain are more commonly associated with myocardial infarction than in men, contributing to a greater diagnostic challenge [12].

Beyond gender issues, there are differences in risk factor distribution and disease management across ethnicities. For example, African American women have a higher rate of coronary heart disease and cardiovascular disease, which constitutes a higher fraction of deaths in this group compared to other minorities [13]. Further, while the metabolic syndrome represents an increased risk for heart disease across Caucasian-African American ethnicity, the individual components of the syndrome is distributed differently [14].

Differences in cardiovascular disease manifestation and presentation, risk factor assessment, pathophysiologic factors, and therapeutic strategies across gender have not been fully addressed. To resolve these remaining challenges, progress across the entire translational spectrum from basic science to population intervention(s) is required. Educational and research infrastructure initiatives such as the NIH "Building Independent Research Careers in Women's Health" (BIRCWH) and related CTSA programs represent excellent opportunities to prepare a new generation of researchers with a rich array of tools to help resolve these important issues.

\section{Integrated Training in Translational Science-Focus on Women's Health}

The CTSA program has created an integrated academic home for clinical and translational science with the resources and infrastructure to train and advance multiand inter-disciplinary investigators and research teams. The UC Davis CTSC Research Education, Training, and Career Development program is committed to a comprehensive approach to translational research training that provides scholars with a rich array of career development opportunities through program curricula, mentored research training, and partnerships with other programs, departments, and institutions. We aspire to transform clinical and translational research training and career development across UC Davis by serving as a resource for best practices, including sharing the many resources created through the CTSC. A strong pipeline and the recognition of a research training culture are clearly emerging, and the CTSC serves as a clinical and translational "research incubator" that incorporates education, clinical and translational resources, and informatics to facilitate novel research initiatives and promote gains in cardiovascular disease prevention (Fig. 1).

The UC Davis BIRCWH program is a key CTSC partner in achieving the goals of team-based training. The overarching objective of the BIRCWH program is to provide junior faculty with state-of-the-art multidisciplinary training that will ensure that they establish independent biomedical research careers in areas relevant to women's health. A second objective of this program is to create an environment that nurtures non-traditional cross-disciplinary collaborations in focused and interactive research areas that are essential to improving the health of women, such as the role of sex hormone levels and hormone replacement therapy for cardiovascular health, cancer risk factors, and 
Fig. 1 UC Davis CTSC research incubator used as a model to illustrate the translational impact on studies on women's cardiovascular health. BIRCWH Building Independent Research Careers in Women's Health, WCMP Women's Cardiovascular Medicine Program, PCIR Participant and Clinical Interactions Resources

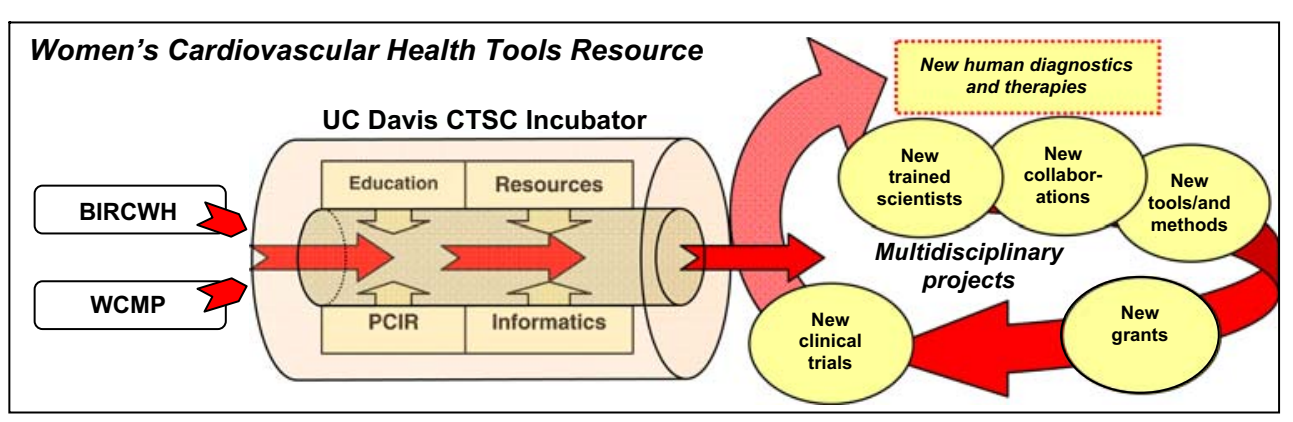

bone health and the use of relevant animal models for translational and preclinical assessments. From its inception, the UC Davis BIRCWH program has focused on four interacting areas of women's health, three of which are highly significant in the prevention of cardiovascular disease: (1) metabolic and nutrition-related syndromes and their repercussions for women; (2) cardiovascular science and its relationship to gender; and (3) lifespan biology and transitions, such as early development, adolescence, and menopause, that bring unique risks to females.

Through the partnership between the BIRCWH and the CTSC training programs, an integration of curricula has brought together clinical and basic science faculty, graduate students, and medical students to work together as teams and to learn from each groups' perspectives and experiences. As a result of these program experiences, scholars are learning by working with colleagues in different disciplines, with different research approaches, and at different career stages. Bringing together scholars from different career stages and disciplines has enriched these learning experiences for all groups by presenting career role models for junior scholars, providing mentoring opportunities for senior scholars, and exposing all scholars to unique and diverse perspectives. Examples of such interactions include CTSC scholars pursuing research projects that focus on risk factor assessment and hormonal pathophysiology in women's cardiovascular disease. Two research projects investigate the roles of endogenous sex hormones (1) in the risk of stroke and on the metabolic syndrome as a risk factor across gender and ethnicity and (2) in the risk of vascular dementia and in conjunction with abnormal cerebral imaging in older affected adults. According to recent clinical trials, women taking exogenous menopausal hormones have an increase in the risk for strokes and no clear coronary heart disease benefit, for currently unknown reasons. The role of endogenous sex hormones in cardiovascular disease in older women is not well studied and might contribute substantially to understanding these findings and to determining women's health overall. Certain animal models such as the nonhuman primate model where reproductive features directly parallel humans provide unique opportunities to address this relationship and provide a greater understanding of the menopausal transi- tion and impact on healthy aging. CTSC scholars are examining prospectively the roles of endogenous circulating levels of estrogens and androgens and of genetic variations within the sex hormone metabolic pathways in the development of stroke, coronary heart disease, and components of the metabolic syndrome. CTSC scholars aim to use newer methods to incorporate the metabolic interplay between estrogens and androgens in their investigations. They are comparing these relations between postmenopausal women and age-comparable men of different ethnicities since differences in sex hormone metabolism could explain in part the gender and ethnic differences in cardiovascular disease.

Another project example involves determining the relation between endogenous sex hormones and abnormal cerebral imaging in older women, compared with older men, who have mild cognitive dysfunction or dementia due to cerebrovascular disease. This prospective cohort study resulted directly from an annual CTSC retreat that fostered new collaborations between a CTSC scholar and members of the UC Davis' Alzheimer's Disease Center, including experts in neuropsychology, pathology, and neuroimaging.

These investigations implement the translational tools of molecular and genetic epidemiology in large prospective population studies. This strategy requires cross-disciplinary collaborations between scholars, trained in endocrinology and epidemiology, and experts in reproductive hormone assays, genetic epidemiology, biostatistics, neuroimaging, and neuropsychiatry. It fosters regular mentoring by CTSC scholars of graduate students and medical fellows working on such projects. This approach will be a key to scientific advancement in understanding gender and ethnic differences in cardiovascular disease and identifying important hormonal differences in the pathogenesis of cerebrovascular disease and of coronary heart disease.

\section{Prevention-Enhancement Through Translational Approaches}

From the inception of the CTSC, the program has focused on providing key support functions to investigators in order to enhance study initiation, increase the quality and 
quantity of support staff availability, and to add new capabilities. An example of this approach is the support provided from the UC Davis CTSC to the UC Davis Women's Cardiovascular Medicine Program. The overarching goal of this program is to provide women with seamless, state-of-the-art, multidisciplinary cardiac health services that comprehensively address the cardiovascular needs of women. The UC Davis Women's Cardiovascular Medicine Program is focusing on five interactive components of cardiovascular health: (1) education and awareness of heart disease as the leading killer of women; (2) screening and risk assessment to address the greater relative risk of major risk factors for women; (3) diagnostic testing and treatment with respect to gender; (4) lifestyle modification and cardiac rehabilitation and its importance to cardiovascular risk reduction; and (5) tracking and evaluation, assessing success, and results for women. Importantly, the Women's Cardiovascular Medicine Program has demonstrated expertise in developing a regional model of women's cardiovascular healthcare serving women in underserved areas, including rural northern California and the central valley. The research activities in the program have been supported by the CTSC, resulting in increased opportunities to undertake community-based research studies due to the infrastructure provided by the CTSC, as well as an ability to serve as coordinating center for multicenter studies, as outlined below.

Another example of CTSA support for studies related to women's health are several investigations on nutritionally based therapies for polycystic ovary syndrome undertaken in the CTSC Clinical Research Center and supported by CTSC informatics and biostatistics resources. The CTSC support has allowed detailed mechanistic studies aimed at cardiovascular risk factor intervention, including blood lipid levels and the degree of insulin resistance $[15,16]$.

\section{Informatics-A Key to Improved Prevention}

Another significant component of the CTSA program is the development and use of innovative research tools and information technologies that promote application of new knowledge and techniques to patient care. An example is the support provided by the CTSC to the Women's Cardiovascular Medicine Program as part of an award from the US Department of Health and Human Services Office on Women's Health entitled "Improving, Enhancing, and Evaluating Outcomes of Comprehensive Heart Care Programs in High-Risk Women". The CTSC partnership made it possible for the UC Davis program to function as a datacoordinating center for six geographically disperse sites, including four academic health centers, one clinical practice center, and one mobile community-based healthcare deliv- ery program [17]. The purpose of the study was to evaluate cardiovascular disease prevention, knowledge, risk awareness, and clinical outcomes in high-risk women and to compare outcomes at 6 and 12 months of patient follow-up. The intervention involved patient education and the application and utilization of the recently published American Heart Association guidelines on "EvidenceBased Guidelines for Heart Disease Prevention in Women" [3]. Each participating site collected patient demographic, survey, and clinical data on over 1,300 women during a 2-year period, involving collection of de-identified patientspecific data from the six sites. These efforts illustrate the synergistic potential of the nationwide CTSA program and its opportunities to enrich specific intervention studies addressing complex diseases, such as in the area of gender-specific cardiovascular health and disease.

\section{CTSA Facilitation of Research Initiatives}

Identification of and support for research funding opportunities represent an important mission of CTSAs. The UC Davis CTSC Grant Facilitation Unit has provided the Women's Cardiovascular Medicine Program assistance with several grant proposals. Some examples include (1) the successfully funded proposal to the Department of Health and Human Services for "Improving, Enhancing, and Evaluating Outcomes of Comprehensive Heart Health Care Programs in High-Risk Women" described above; (2) a Women's Career R01 that is pending award notification; and (3) a Challenge Grant application that is part of the American Recovery and Reinvestment Act through the National Institutes of Health. By providing assistance with these proposals, we have been able to help further initiatives and projects aimed at cardiovascular research goals in women's health. Further, the creation of opportunities for a broad spectrum of investigators to participate in translational activities represents an important CTSA mission and represents another example of how the CTSAs can facilitate collaborative research efforts.

\section{Summary}

The CTSA program offers novel and far-reaching opportunities to support clinical and translational research in common and chronic disease areas. The many complexities contributing to the currently distant goal of reducing cardiovascular mortality among women suggest that this area of research may in particular benefit from synergistic interactions with the national CTSA programs. It is apparent that the UC Davis CTSC has substantially impacted clinical and translational research to a significant 
extent at our institution, and, in the first 2 years, has markedly transformed the ease and ability of UC Davis researchers to engage in clinical and translational research. Here, we have provided some examples that highlight the potential of the program in its abilities to support, extend, and enrich efforts to increase our understanding of and ability to prevent cardiovascular disease in women.

Acknowledgments The project was supported by the UC Davis Clinical and Translational Science Center (RR 024146); Dr. Lee is a CTSC K12 awardee (RR 024144).

Open Access This article is distributed under the terms of the Creative Commons Attribution Noncommercial License which permits any noncommercial use, distribution, and reproduction in any medium, provided the original author(s) and source are credited.

\section{References}

1. Thom, T., Haase, N., Rosamond, W., et al. (2006). For the American Heart Association Statistics Committee and Stroke Statistics Subcommittee. Heart Disease and Stroke Statistics2006 update. Circulation, 113, e85-e151.

2. Loomba, R. S., \& Arora, R. (2008). Prevention of coronary heart disease in women. Therapeutic Advances in Cardiovascular Disease, 2, 321-327.

3. Mosca, L., Banka, C. L., Benjamin, E. J., Berra, K., Bushnell, C., Dolor, R. J., et al. (2007). Evidence-based guidelines for cardiovascular disease prevention in women: 2007 Update. Journal of the American College of Cardiology, 49, 1230-1250.

4. Mosca, L., Linfante, A. H., Benjamin, E. J., et al. (2005). National study of physician awareness and adherence to cardiovascular disease prevention guidelines in the United States. Circulation, 111, 499-510.

5. Zerhouni, E. A. (2005). US biomedical research. Basic, translational, and clinical sciences. JAMA, 294, 1352-1358.

6. Zerhouni, E. A. (2006). Clinical research at a crossroads: the NIH roadmap. Journal of Investigative Medicine, 54, 171-173.
7. Asmuth, D., Wun, T., Mullen, N., Garcia, E., Chedin, E., Whitney, E., et al. (2009). UC Davis Clinical and Translational Science Center: Coming of age. Clinical Translation Science, 9, 98-101.

8. Berglund, L., \& Tarantal, A. (2009). Strategies for innovation and interdisciplinary translational research: removal of barriers through the CTSA mechanism. Journal of Investigative Medicine, 57, 474-476.

9. Bittner, V. (2006). Perspectives on dyslipidemia and coronary heart disease in women: an update. Current Opinion in Cardiology, 21, 602-607.

10. Wilson, P. W. F., Smith, S. C., Blumenthal, R. S., Burke, G. L., \& Wong, N. D. (2003). Selecting patients for atherosclerosis imaging. Journal of the American College of Cardiology, 41, 1898-1906.

11. Pischon, T., Hu, F. B., Rexrode, K. M., Girman, C. J., Manson, J. E., \& Rimm, E. B. (2008). Inflammation, the metabolic syndrome, and risk of coronary heart disease in women and men. Atherosclerosis, 197, 392-399.

12. Bedinghaus, J., Leshan, L., \& Diehr, S. (2001). Coronary artery disease prevention: what's different for women. American Family Physician, 63, 1393-1400.

13. Mosca, L., Ferris, A., Fabunmi, R., \& Robertson, R. M. (2004). Tracking women's awareness of heart disease in America. Circulation, 109, 573-579.

14. Anuurad, E., Chiem, A., Pearson, T. A., \& Berglund, L. (2007). Metabolic syndrome components in African Americans and European-Americans patients and its relation to coronary artery disease. American Journal of Cardiology, 100, 830-834.

15. Kasim-Karakas, S. E., Cunningham, W. M., \& Tsodikov, A. (2007). Relation of nutrients and hormones in polycystic ovary syndrome. American Journal of Clinical Nutrition, 85, 688694.

16. Kasim-Karakas, S. E., Almario, R. U., \& Cunningham, W. (2008). Effects of protein versus simple sugar intake on weight loss in polycystic ovary syndrome (according to the National Institutes of Health criteria). Fertility and Sterility, epub.

17. Villablanca, A. C., Baxi, H., \& Anderson, K. (2008) Novel data interface for evaluating cardiovascular outcomes in women. Handbook of Research on Information Technology Management and Clinical Administration in Healthcare. In A. Dwivedi (Ed.). Hershey: IGI (in press). 